\title{
Learning From Interactive Whiteboard Instruction Technology in Teaching Students With Autism Spectrum Disorders
}

\author{
Fayez S.Maajeeny ${ }^{1}$ \\ ${ }^{1}$ Department of Special Education, University of Jeddah, Jeddah, Saudi Arabia \\ Correspondence: Fayez S. Maajeeny, Department of Special Education, University of |Jeddah, Jeddah, Saudi \\ Arabia. Tel: 966-544-054-505.E-mail: fmaajeeny@uj.edu.sa
}

Received: October 1, 2018 Accepted: February 28, 2019 Online Published: March 12, 2019

doi:10.5539/gjhs.v11n4p19 URL: https://doi.org/10.5539/gjhs.v11n4p19

\begin{abstract}
Interactive whiteboard instruction technology provides interactive learning environment and serves as a motivational tool for the students. The study aims to investigate the effectiveness of interactive whiteboard (IAW) to teach early numeracy skills to the ASD students. The study has employed single-case design methodology and evaluated students for the effectiveness of using interactive whiteboard for teaching skills to the students through multiple probe design. A total of five baseline sessions were conducted on total four recruited students. During the intervention, data was obtained for at least three sessions from the date each student reached the acquisition criteria. The results showed that introduction of the intervention resulted in all participants meeting the established criteria. The early numeracy skills were generalized by all the four students to a different setting and with different materials. These results have supported the effectiveness of the interactive whiteboard, coupled with DTT to teach early numeracy skills to students with ASD. The study has concluded that interactive whiteboard with DTT was effective to teach early numeracy skills to the ASD students.
\end{abstract}

Keywords: learning, interactive whiteboard, technology, students, autism spectrum disorders

\section{Introduction}

There is dramatic increase in the prevalence of Autism Spectrum Disorder (ASD). The primary deficits experienced by these individuals include communication and social interactions. Moreover, the individuals suffering from ASD may even exhibit repetitive behaviours. The learning and educational performance of ASD individuals is affected, negatively. As a result of this negative impact, individuals experience difficulty in learning and performance academic skills such as numeracy skills. These individuals are likely to suffer from decreased academic performance, poor level of self-confidence, and inability to perform daily tasks (Estes et al., 2011). Therefore, there is a need of educational reforms focusing on the education of students' inclusive setting and meeting due to increase in ASD prevalence.

It is important for the educators to understand their strength and needs to provide appropriate services to the individuals suffering from ASD for developing and implementing effective individualized instruction for their students. A study conducted by Constable et al. (2013) confirmed the importance of developing effective tools and interventions to facilitate ASD individuals by the educational professionals. A recent report published by CDC in 2014 narrated that individual of any race, ethnicity, and socioeconomic status is likely to get affected by ASD (Centres for Disease Control and Prevention, 2014). ASD has been classified as a neurological disorder that disturbs brain functioning, which is diagnosed between 18 months to three years of age (National Institute of Mental Health, 2012). The American Psychiatric Association (2013) stated that there is flexibility in the behaviour of individuals with first level of severity. However, this may cause significant interference with normal functioning of the individual in one or more context. These individuals may even face problems of organizing and planning, which obstructs their independence (American Psychiatric Association, 2013).

Substantial support is needed by the individuals, who are diagnosed with second level of severity and they show deficits in verbal and non-verbal social communication skills. There is increase in abnormal responses to social interactions and limited initiation of social interactions among the individuals with ASD. These individuals react different in normal daily routine as they find difficulty in coping up with environmental changes, inflexible behaviour, and different actions (American Psychiatric Association, 2013). The developmental progress of individuals diagnosed with ASD is affected, negatively. Deficits in joint attention skills have a major impact on the 
acquisition of early skills, which make it difficult to demonstrate receptive and expressive language and engage in everyday human interactions for individuals with ASD (Egel, 2012).

The use of interactive whiteboards (IAWs) is considered as a well-established educational technological innovation around the world. Initially, these whiteboards were created for the office environment; however, in recent times they tend to represent a new learning technology for the students within their classrooms. They provide interactive learning environment and serve as a motivational tool for the students. The features rendered by these whiteboards play an important part towards the development of effective interventions for normal as well as disabled individuals (Maajeeny, 2017). Therefore, the present study has supported the utilization of different instructional procedures that include the use of technology and reinforcement for the students with ASD.

There are only few studies that have examined IAW to teach early numeracy skills to ASD students. The present study tends to demonstrate different results from the previous studies as it has mainly targeted the generalization, acquisition, and maintenance of student's skills. Therefore, the present study aims to assess the learning capabilities of students with ASD through IAW instruction technology.

\section{Material and Methods}

\subsection{Study Design}

A single-case design methodology was used that aims to assess the impact of intervention package across 4 students with ASD. The participants were evaluated for the effectiveness of using IAW for teaching skills to the students through multiple probe design.

\subsection{Study Procedure}

The study has measured acquisition, generalization, and maintenance of skills as a result of the interactive whiteboard intervention. Specifically, the baseline condition was an assessment of the skills selected to be taught, namely counting with one-to-one correspondence and representation of numbers (i.e., understanding that a number refers to an item or a set of items). There were five baseline sessions conducted on each of the four participants. First student was selected randomly to receive the intervention given stable and low-level baseline results under 50\% for all participants. A baseline probe was collected for Students 2, 3, and 4, when the first student reached the criteria of $60 \%$ accuracy or better for one session during the intervention. Later, other students were also made to receive the intervention. An additional three baseline sessions were collected to establish a trend and level to ensure that the second student's performance remained under $50 \%$. The same sequence of baseline procedures was repeated for Student 3 and Student 4 when the criterion of $60 \%$ accuracy or better for one session was reached by the preceding participant during the intervention condition. The students were exposed to the use of IAW to learn skills for the first time in one-on-one instruction.

\subsection{Data Collection and Analysis}

The main focus of the study was on the teacher's activity system, where the IAW is considered as a pedagogical tool. The specific skills targeted during the investigation were counting with one-to-one correspondence with numbers 1-5 or 6-10 and representation of numbers. IAW technology was used as a tool to achieve his or her pedagogical goal and incorporate it into the activity in a pedagogically sound manner. The data was obtained for at least three sessions from the date each student reached the acquisition criteria during the intervention condition. Additionally, $50 \%$ of all intervention sessions were scored from the videotape for procedural reliability using a rubric that included each step of the intervention to measure the procedural consistency with which the intervention was implemented.

\section{Results}

The study has helped in understanding the effectiveness of using IAW in teaching ASD students. Student's engagement was observed in using IAW for analysing their potential affordances. The results have been presented in relevancy with each condition displayed across the four participants. Figure 1 has illustrated the impact of using IAW and discrete trial training (DTT) for teaching early numeracy skills (one-to-one correspondence and representation of numbers). However, the progress in performance of each student has been illustrated below;

\subsection{Student 1}

Figure1 has shown that the baseline data for student 1 was collected on five sessions (1-5) with low level responding and the average during baseline was $24 \%$ (ranging between $20 \%$ - $40 \%$ ). The instruction condition was introduced for five sessions ( 6 through 10) on session 6 . In the instruction condition, student 1 had changes in both trend and the level of responses with a mean of $68 \%$ correct responses (ranging between $40-100 \%$ ) until she mastered the criteria on sessions $8-10$ by achieving $80 \%$ or better for three consecutive sessions. The targeted early 
numeracy skills were measured using the IAW but without prompting that helped in collecting post-intervention probes on three consecutive sessions following instruction (11 through 13). Student 1's mean for correct responses was $93 \%$ (ranging between $80 \%-100 \%$ ) for three consecutive post-intervention sessions.

Generalization probes for Student 1 were collected for one session during baseline and two sessions immediately after the post-intervention probes without the IAW. Student 1's correct responses during the baseline generalization probe was $20 \%$ that increased after the post-intervention probes to an average of $80 \%$ correct (ranging between $60 \%-100 \%$ ). After the generalization probes on the 20th, 27th, 29th, 33rd, and 37th session, the maintenance data was collected using the IAW on five sessions conducted and the mean was $100 \%$ correct. Overall, visual inspection of the data showed that student 1 had a stable trend with a low level of responding during baseline. Moreover, student 1 had a level change compared to the baseline when the instruction was introduced and she established an ascending trend within the 8th session. Student 1's data showed an increasing change in the level of responding from the baseline condition for the post-intervention probes, generalization, and maintenance conditions.

\subsection{Student 2}

Student 2's baseline data were collected on nine sessions (1 through 11) with stable and low level responding, with a mean of $27 \%$ (ranging between $20 \%-40 \%$ ) correct. On session 12 , the instruction condition was introduced using the IAW on six sessions (12 through 17). Student 2's behavior changed in both trend and the level with a mean of $57 \%$ (range, $20 \%-100 \%$ ) correct responses in the instruction condition. Post-intervention probes were collected on three consecutive sessions (18 through 20) to measure the early numeracy skills post-instruction using the IAW without prompting. Student 2's mean for correct responses was $87 \%$ (ranging between $80 \%-100 \%$ ). Generalization probes were collected without using the IAW on one session during baseline and two sessions immediately after the post-intervention probes condition. Student 2's correct responses during the baseline generalization probe was $20 \%$ that increased after the post- intervention probes to an average of $90 \%$ correct responses (ranging between $80 \%-100 \%$ ). The maintenance data was collected using the IAW on the 27th, 29th, $33 \mathrm{rd}$, and 37th session on three sessions and the mean was 95\% correct responses (ranging between $80 \%-100 \%$ ).

A stable trend with low level responding during baseline was depicted through the visual inspection of the student 2 's data. Student 2 initially had no level change two sessions after the baseline when the instruction was introduced; however, she established an ascending trend within the 14th session. Student 2's data showed an increasing change in the level of responding from the baseline condition for the post-intervention, generalization, and maintenance.

\subsection{Student 3}

Baseline data for student 3 was collected on 10 sessions (1 through 17) that was stable with low level responding. Student 3's mean during baseline was $22 \%$ (ranging between $20 \%-40 \%$ ) correct. On session 18 , the instruction condition was introduced and data were collected on three sessions (18 through 20). Student 3 had immediate changes in both trend and the level during the instruction condition. The mean for the three sessions of instruction was $87 \%$ (ranging between $80 \%-100 \%$ ) correct. Post-intervention probe data was collected on three consecutive sessions (21 through 23) using the IAW without prompting to measure the early numeracy skills post-instruction. Student 3's mean for the post-intervention probes was 93\% (ranging between $80 \%-100 \%$ ) that was correct for 3 consecutive probe sessions.

IAW on one session during baseline and two sessions immediately after the post-intervention probe condition were used for the collection of generalization probes. Student 3's correct responses during the baseline generalization probe was $20 \%$ and increased after post-intervention probes to an average of $80 \%$ correct across the two generalization sessions. Maintenance data was collected using the IAW on the 27th, 29th, 33rd, and 37th session on four sessions. The mean was 95\% correct responses (ranging between $80 \%-100 \%$ ). The visual inspection of the data showed that during baseline student 3 had a stable trend with low level responding. When the instruction was introduced, student 3 changed over the baseline level immediately. However, student 3's data showed change with increasing levels of responding from the baseline condition for the other three conditions.

\subsection{Student 4}

The baseline data for student 4 was collected on 11 sessions (1 through 21) with an average of $14 \%$ (ranging between $0 \%-20 \%$ ) correct. The data was collected during instruction, when the early numeracy skills were taught using the IAW on ten sessions (22 through 31). The mean for the 10 sessions of instruction was $52 \%$ (ranging between $0 \%-100 \%$ ) correct. Post-intervention data probes were collected on three consecutive sessions (31 through 33) using the IAW without prompting to measure the early numeracy skills post-instruction. For 3 consecutive probe sessions, the mean for correct responses for student 4 was $66 \%$ (ranging between $60 \%-80 \%$ ). 
The generalization probes were collected without the IAW on one session during baseline and two sessions immediately after the post-intervention probe condition. The correct response during the baseline generalization probe for student 4 was $20 \%$, which increase after the post-intervention probes to an average of $40 \%$ correct. Maintenance data were collected on one session using the IAW on the 37 th session with $60 \%$ correct. Therefore, it can be stated that visual inspection of the data of student 4 showed that he had a stable trend with low level responding during baseline. Moreover, student 4 displayed variable data that ranged from $0 \%$ to $100 \%$ correct after introducing the instructions. There was no change in the level compared to the baseline; however, he had established a clear pattern of an ascending trend during instruction. For the other three conditions, Student 4's data showed a change in responding from the baseline condition to post-intervention, generalization, and maintenance conditions.

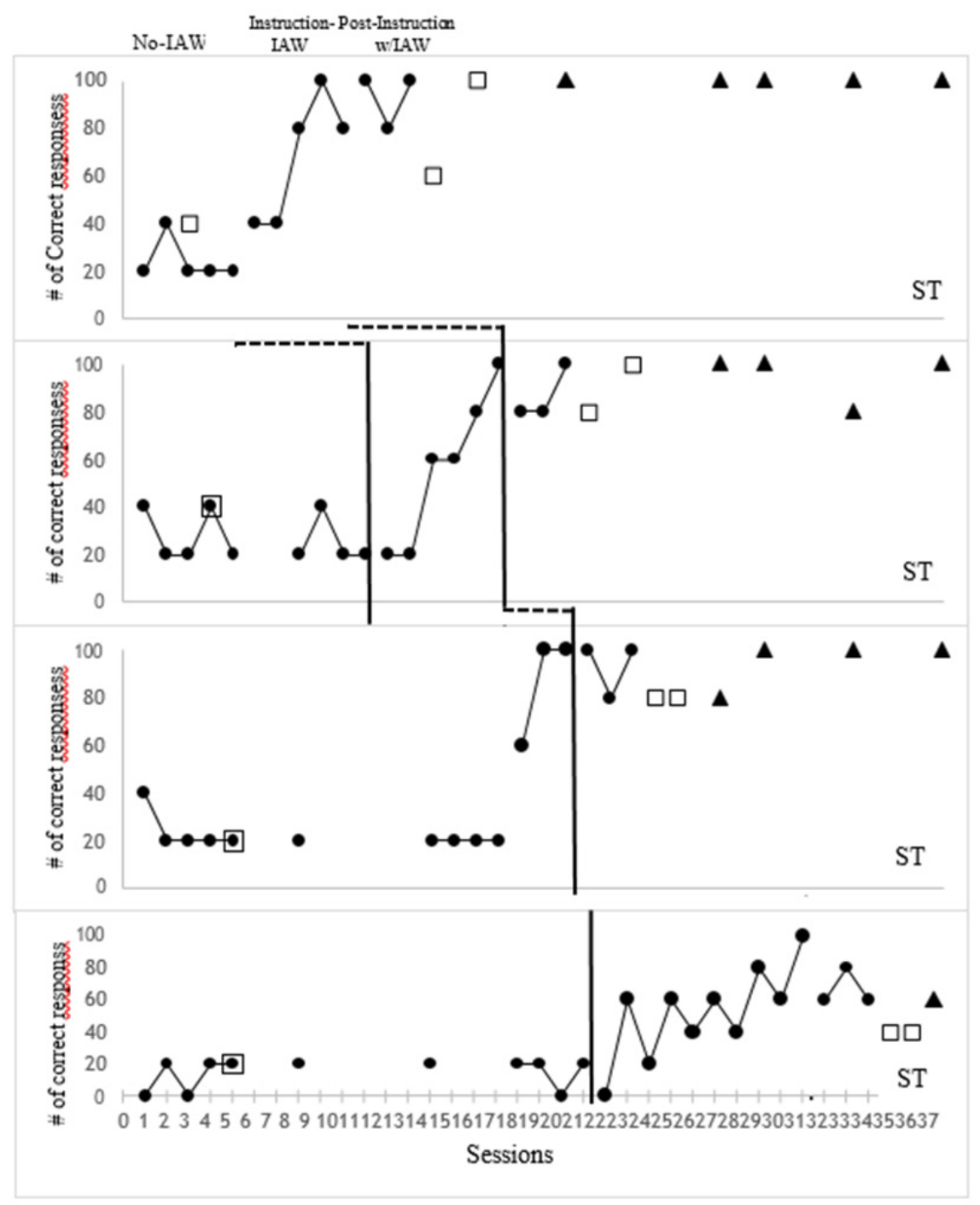

Figure 1. Effects of the IAW on Early Numeracy Skills across Students 1, 2, 3, and 4

The percent of non-overlapping (PND) data points between each condition and the means across all participants were calculated to estimate the effect size of the intervention and support the conclusion about the visual inspection of the data.

\section{Discussion}

The results have clearly shown that IAWs are effective in teaching early numeracy skills to the ASD students. The results also showed that high levels of accuracy are to be achieved through the instructional sessions. Similar to the results of present study, another study conducted by Yakuova \& Taber-Doughty (2013) stated that implementation 
of IAW technology is effective in one-to-one instructional situations for teaching early numeracy skills to the ASD students. The integration of IAW with DTT has proved to be effective to teach early numeracy skills to ASD students as these children suffer from major difficulties in the area of language, emotional, social, and cognitive skills. In the present study, IAW technology was effective in teaching early numeracy skills to the four ASD students; however, student 4 took longer time to reach the instructional criteria.

According to the results of the present study, targeted early numeracy responses were learnt by all the four participants as they reached the mastery criteria. For three consecutive sessions, three ASD students performed within the mastery criteria at a very high level ranging from $80 \%$ to $100 \%$. Whereas, the performance of fourth student was lower as compared to other to reach $100 \%$ for one of the sessions. Previous studies demonstrating the effectiveness of IAW in teaching letter sounds (Campbell \& Mechling, 2009), sight words (Mechling et al., 2008), and skills related to daily life (Yakuova \& Taber- Doughty, 2013) have been extended in the light of the findings of this study. However, results of the present study are extensive as they are based on teaching early numeracy skills to young elementary school students (kindergarten and first graders).

During the intervention session, the introduction of IAW helped the students in reaching the desired criteria of $100 \%$ accuracy for one session or $80 \%$ or better for three consecutive sessions. Similar results were depicted by Mechling et al. (2008). The study compared flash card instruction to instruction using IAW and the results clearly depicted that the performance of students was improved using IAW. The students recruited in the study conducted by Mechling et al. (2007) participated in pretest/posttest, instruction, and generalization conditions; whereas, in the present study all the four students participated in baseline, intervention, post-intervention probes, generalization, and maintenance conditions. The results proposed by Mechling et al. (2007) showed no obvious preferences or difference as participants in all studies including the current study reached a high level of correct responding during the intervention condition using the IAW; although, the study had used different instructional procedures and instructional arrangements.

Multisensory is among the key variables that help students in reaching the desired criteria within short periods of time using IAW technology. This approach is likely to be developed using SMART Notebook collaborative learning software that enable students to experience interactive activities during instruction, hear auditory and see visual feedback, allow them to touch the screen while counting the displayed pictures, and receive visual feedback for correct responses or incorrect responses. A study conducted by Keay-Bright (2011) observed that collaborative learning software contributed to students' learning of early numeracy skills. Moreover, the results also showed that stressed tactile interaction could play an important role in enhancing sensory experiences. Another study conducted by Murray et al. (2005) depicted that presence of interactive environment with sensory simulation is important while teaching early numeracy skills to the ASD students.

A similar study conducted by Odom et al. (2015) assessed the use of IAW as cognitive tools for teaching and learning among ASD students. The results depicted that IAW is likely to provide ASD students with opportunities to learn in a format that supports their visual modality. Moreover, it also provides a controlled and predictable visual format for students to manipulate and master. This is likely to be a positive approach for the development of learning skills among the ASD students (Odom et al., 2015). Similar results to the present study were deduced by Stanley (2017) as the study examined the impact of IAW during reading instruction on student engagement and achievement among ASD students. The results depicted that no noticeable differences in achievement or engagement between the two methods of intervention for ASD students (Stanley, 2017).

\section{Conclusion}

The present study has assessed the learning capabilities of ASD students through IAW instruction technology. It has mainly focused on using the IAW to teach early numeracy skills for ASD students. The strategy used in this study is effective to teach other academic and more complex skills to the students. However, several limitations have been reported despite of showing IAW technology as an effective method for teaching of early numeracy skills to ASD students. The ability to generalize target responses by each student to different setting was conducted by only one instructor, and generalization was only assessed within the same classroom but in a different area of the classroom. This did not allow the researcher to determine the difference in participants' responses to other instructors, different school settings, and novel material. Moreover, it is possible that reinforcement may have affected the rate of students' responses during each condition. The intervention of the current study was conducted only in the kindergarten classroom, which required the investigator to pick up and drop off Student 2 and Student 3 from their first grade classroom. Transition of students to a new environment was not considered while interpretation of the results. Therefore, future study needs to be conducted in the setting where conditions approximates the normal environment and minimizes the opportunity with naturally occurring distractions. 


\section{Acknowledgements}

The author is very thankful to all the associated personnel in any reference that contributed in/for the purpose of this research. Further, this research holds no conflict of interest and is not funded through any source.

\section{Competing Interests Statement}

The authors declare that there are no competing or potential conflicts of interest.

\section{References}

American Psychiatric Association. (2013). Diagnostic and statistical manual of mental disorders: DSM-5. Washington, DC: American Psychiatric Association.

Campbell, M. L., \& Mechling, L. C. (2009). Small group computer-assisted instruction with SMART Board technology: An investigation of observational and incidental learning of nontarget information. Remedial and Special Education, 30(1), 47-57. https://doi.org/10.1177/0741932508315048

Centers for Disease Control and Prevention. (2014). Prevalence of autism spectrum disorders among children aged 8 years: Autism and developmental disabilities monitoring network, 11 sites, United States, 2010. MMWR Surveillance Summaries, 63(2), 1-22.

Constable, S., Grossi, B., Moniz, A., \&; Ryan, L. (2013). Meeting the Common Core State Standards for Students with Autism. TEACHING Exceptional Children, 45(3), 6-13. https://doi.org/10.1177/004005991304500301

Egel, A. L. (2012). Introduction to autism spectrum disorders. In A. L. Egel, K. C. Holman, \&amp; C. H. Barthold (Eds.), School success for kids with autism (pp. 7-18). Waco, TX: Prufrock Press, Inc.

Estes, A., Rivera, V., Bryan, M., Cali, P., \&; Dawson, G. (2011). Discrepancies between academic achievement and intellectual ability in higher-functioning school-aged children with autism spectrum disorder. Journal of Autism and Developmental Disorders, 41(8), 1044-1052. https://doi.org/10.1007/s10803-010-1127-3

Keay-Bright, W. (2012). Designing for playfulness: Investigating the therapeutic potential of technology. University of Wales. Retrieved from http://hdl.handle.net/10369/3263

Maajeeny, F. (2017). The Effects of Interactive Whiteboard Instruction on Early Numeracy Skills of Students with Autism Spectrum Disorders (Doctoral dissertation).

Mechling, L. C., Gast, D. L., \& Krupa, K. (2007). Impact of SMART Board technology: An investigation of sight word reading and observational learning. Journal of Autism and Developmental Disorders, 37(10), 1869-1882. https://doi.org/10.1007/s10803- 007-0361-9

Mechling, L. C., Gast, D. L., \& Thompson, K. L. (2008). Comparison of the effects of SMART Board technology and flash card instruction on sight word recognition and observational learning. Journal of Special Education Technology, 23(1), 34- 46. https://doi.org/10.1177/016264340802300103

Murray, D., Lesser, M., \& Lawson, W. (2005). Attention, monotropism and the diagnostic criteria for autism. Autism, 9(2), 139-156. https://doi.org/10.1177/1362361305051398

National Institute of Mental Health. (2012).

Odom, S. L., Thompson, J. L., Hedges, S., Boyd, B. A., Dykstra, J. R., Duda, M. A., ... \& Bord, A. (2015). Technology-aided interventions and instruction for adolescents with autism spectrum disorder. Journal of autism and developmental disorders, 45(12), 3805-3819. https://doi.org/10.1007/s10803-014-2320-6

Stanley, N. (2017). Effects of interactive whiteboard technology on the achievement and engagement of elementary-aged students with high-functioning Autism Spectrum Disorder in the content of reading (Doctoral dissertation, Colorado State University. Libraries).

Yakubova, G., \& Taber-Doughty, T. (2013). Brief report: Learning via the electronic interactive whiteboard for two students with autism and a student with moderate intellectual disability. Journal of Autism and Developmental Disorders, 43(6), 1465-1472. https://doi.org/10.1007/s10803-012-1682-x

\section{Copyrights}

Copyright for this article is retained by the author(s), with first publication rights granted to the journal.

This is an open-access article distributed under the terms and conditions of the Creative Commons Attribution license (http://creativecommons.org/licenses/by/4.0/). 